Many of the contributors wrote from a UK perspective, which was more evident in chapters involving local laws such as the chapters on Neuropsychology and the Law and Mental Capacity. Nevertheless, these and all other chapters were very informative and of very high quality.

Chapter 6 by Crawford is an excellent reminder of the level of quantitative measurement theory that separates a clinical neuropsychologist from a clinical psychologist. Although this chapter also provides an excellent overview of the basics, it includes a discussion of the importance of incorporating information beyond mere error in measurement when interpreting test scores; specifically, base-rates of impaired scores and the complexity of interpreting differences between scores. Moreover, the discussion of measurement of change over time appears universally helpful, but a brief discussion of reliable change indices corrected for practice effects may have tempered the argument for regression-based formulae when interpreting declines or improvement over time. Another chapter that may be of universal interest is the thoughtful discussion of how apathy interacts with depression and with cognitive testing detailed by Brown in Chapter 4. Chapter 22 by Tyerman and King on interventions for psychological problems after brain injury includes an excellent and practical discussion of how psychotherapy may need modification based on specific neuropsychological impairments.

Overall, this highly diverse book is comprehensive in its brief review of theory, assessment, and intervention related to a variety of cognitive difficulties. Given the brevity of the theoretical background this book is likely better aimed at those with postgraduate training in clinical psychology or those with medical training.

Megan E. O'Connell University of Saskatchewan Saskatoon, Saskatchewan, Canada

EPILEPSY IN CHILDREN AND AdDOLESCENTS. 2013. Edited by James W. Wheless, David F. Clark, Amy L. McGregor, Phillip L. Pearl, Yu-Tze Ng. Published by Wiley-Blackwell. 378 pages. C $\$ 130$ approx.

\section{Rated tristis}

A short, readable book about Epilepsy in Children is a big challenge to write but this one is reasonably successful. The literature is enormous. A PubMed search in 2013 of "Epilepsy in children" yielded 37,568 references! The potential audience is varied for a book such as this. Epilepsy experts are probably most interested if a new topic is reviewed in depth, general pediatric neurologists if there is an overview, general pediatricians if the discussion is very practical, neurology residents and medical students if it looks like a place to start. Families are of course intensely interested but do they read text books? Dr. Wheless has an ambitious agenda - "I envisage this book to be a resource for all physicians and other professionals taking care of children with seizures or epilepsy." The goal was for each chapter to be succinct, so a physician confronted with a child who has seizures would have an efficient resource for answering questions and designing treatment". The book is organized into six sections Epidemiology and Classification, Diagnostic Evaluation, Principles of Treatment, Generalized seizures and syndromes, Focal seizures and syndromes, and Epilepsies relative to age, etiology or duration. This last section includes three comprehensive chapters on neonatal seizures, febrile seizures and status epilepticus. All 25 authors are from the USA.

Before launching into a critique, I need to make two disclosures. First, several of the authors are my professional friends. Second, I have co-edited a recent shorter book about epilepsy in children directed to general pediatricians ${ }^{1}$.

This book struggles (as we all do) with seizure and epilepsy classification. The chapter on Classification discusses and tries to incorporate many of the compelling suggestions for the ILAE organization/classification from $2010^{2}$. However, most of the book continues to use the ILAE 1989 system $^{3}$. This gives rise to some interesting issues. For example, the chapter on Evaluating the Child with Seizures suggests that nearly all patients have an "ILAE" syndrome - this was based on the 1989 classification ${ }^{4}$. The 2010 system allows a syndrome diagnosis for fewer than $40 \%{ }^{5}$. A substantial portion of the book focuses on specific groups of syndromes based on the 1989 classification but many chapters offer an apology for not following the 2010 scheme.

The 20 chapters vary a great deal in the amount of detail offered. Not surprisingly the chapter on Evaluating the Child with Seizures would be most useful to beginners. The excellent introductory chapter on EEG would be most appropriate for neurology residents. The sections on focal epilepsies would not help experts involved in epilepsy surgery, but at least the value of epilepsy surgery is clearly and enthusiastically presented. The chapters on Pharmacology and choice of AEDs are excellent and I especially liked the comparison of recommendations for treatment by experts in different areas of the world (not surprisingly they vary quite a bit). The chapter on when to start and when to stop AEDs is well balanced. Unfortunately, through the book there is very little attention paid to long term psychosocial outcome.

General practical issues of epilepsy care are not heavily emphasized such as the frequency of follow up appointments and the value of repeat EEGs or imaging, although there are excellent tables outlining the indications for therapeutic drug and toxicity monitoring. There are few comments about restrictions for activities (except in uncontrolled absence), alcohol or recreational drug use, contraception for teenagers, monitoring or enhancing compliance, special issues related to schooling or transition to adult care. Mortality is rarely mentioned and the term SUDEP is conspicuously absent from the index. There are few absolutes in childhood epilepsy care but the authors tend to couch them with "wiggle words". For example, the seizure prognosis for Rolandic epilepsy is said to be "quite good" rather than virtually $100 \%$ remission or "breath holding spells often resolve by five to seven 
years of age," even though they are exceedingly rare in school aged children.

In general, I think this book very sound. It would be an excellent introduction for neurology residents and a good review for general pediatric neurologists who feel a bit out of touch with current treatments for epilepsy. I am not sure that general pediatricians would find the content sufficiently directive. Family physicians might be helped by some chapters but in general the slant is more towards specialists. Some medical students will be overwhelmed by the details in many chapters. Epilepsy experts would be interested in the struggles about classification. Lastly, the book is not aimed at families of children with epilepsy.

Peter Camfield Halifax, Nova Scotia, Canada

\section{REFERENCES}

1. Appelton R, Camfield P. Childhood epilepsy: management from diagnosis to remission. Cambridge NY 2011. ISBN 9780521763257.

2. Berg AT, Berkovic SF, Brodie MJ, et al. Revised terminology and concepts for organization of seizures and epilepsies: report of the ILAE Commission on Classification and Terminology, 20052009. Epilepsia. 2010;51:676-85.

3. Commission on Classification and Terminology of the International League Against Epilepsy. Proposal for revised classification of epilepsies and epileptic syndromes. Epilepsia. 1989;30:389-99.

4. Berg AT, Shinnar S, Levy SR, Testa FM, Smith-Rapaport S, Beckerman B. How well can epilepsy syndromes be identified at diagnosis? A reassessment 2 years after initial diagnosis. Epilepsia. 2000;41:1269-75.

5. Wirrell EC, Grossardt BR, Wong-Kisiel LC, Nickels KC. Incidence and classification of new-onset epilepsy and epilepsy syndromes in children in Olmsted County, Minnesota from 1980 to 2004: a population-based study. Epilepsy Res. 2011;95:110-18.

Drug-Induced Neurological Disorders. Third Revised and Expanded Edition. 2012. By K.K. Jain. Published by Hogrefe Publishing. 452 pages. C $\$ 130$ approx.

\section{Rated trirtris}

Practicing clinicians are well aware that adverse effects may occur from the medications they prescribe. Uncommon adverse effects reported by the patient are met sometimes with suspicion or incredulity, and followed up by asking a more experienced colleague, looking it up in the latest version of the CPS or Epocrates, or by simply "googling" it. While those references are certainly helpful, the breadth, scope and perspective can be lacking.

Drug-Induced Neurological Disorders is the 3rd edition of this text by K.K. Jain. The author has a unique background in being trained in both neurology and neurosurgery (including training at several Canadian centres) before practicing neurosurgery for over 20 years. He subsequently became a medical advisor to industry, founded his own Biotech company and is senior associate editor for MedLink Corporation; a recent PubMed search of the author lists nearly 200 publications to his credit.

Dr. Jain focuses on the neurological effects of medical treatments and as such his text is indispensable for the consulting neurologist. In the preface, he reminds us that "many of the wellknown adverse effects are no longer being published and the number of publications is not an indication of the frequency of occurrence of a particular event." He acknowledges being more selective in his references (which are listed at the end of each chapter) compared to previous editions. The book is divided into 27 chapters, with the first nine chapters accounting for nearly half the volume. He begins with epidemiology and clinical significance, followed by a review of pathomechanisms. Chapters on encephalopathies, disorders of consciousness, neuropsychiatric disorders, headaches and seizures follow. All levels of and conditions related to the nervous system are covered, including movement disorders, neuromuscular conditions, aseptic meningitis, idiopathic intracranial hypertension, autonomic disorders and sleep disorders, and even disorders affecting the pituitary system. Rare conditions including eosinophilia myalgia syndrome and subacute myelo-optico-neuropathy, a condition occurring essentially only in Japan, have their own chapter. The book concludes with chapters on adverse effects of biological therapies and neurological complications of anesthesia.

As this is a single authored text, there is a consistent writing style which I found very easy to read. He manages to present complex topics concisely yet appropriately detailed. The figures and tables are clear and useful. While some of the tables are a bit lengthy, the author reinforces which treatments are particularly associated with certain conditions.

The history, mechanisms of actions, clinical presentation, risk factors, pathology, course, prognosis and management of druginduced neurological disorders are all covered in this book. When there is conflicting evidence or opinion, Dr. Jain presents the information before summarizing. The book overall and individual chapters are all well organized, and the fonts and lay-out make it visually appealing despite having no color pictures. After the text concludes, there is an index of drugs, followed by a symptom index. Some things are mentioned more than once, but that is to be expected and does not detract.

I highly recommend this outstanding reference for anyone in independent neurological practice. For residents and those at a university medical centre, an individual copy may not be necessary though I view it as incumbent on your library (or kind colleague) to have this text available for you to borrow as needed.

\section{Alex Rajput \\ Saskatoon, Saskatchewan, Canada}

